\title{
CMMI aplicado en el aula: aprendizaje basado en proyectos
}

\section{CMMI applied in the classroom: project-based learning}

\author{
LÓPEZ-SEGURA, Teresita de Jesús†*, NACHEZ-MARTÍNEZ, Wendy Denisse, TORRES-TINOCO, \\ Anahí Monserrat y SÁNCHEZ-DURÁN, Ricardo Miguel
}

Universidad Tecnológica de León, Área Tecnologías de la Información. Blvd. Universidad Tecnológica \#225 Col. San Carlos. CP. 37670 León, Gto. Mex.

ID 1er Autor: Teresita de Jesús, López-Segura / ORC ID: 0000-0001-5801-952X, Researcher ID: T-6804-2018, arXiv: tlopez\#3, CVU CONACYT ID: 683314

ID 1er Coautor: Wendy Denisse, Nachez-Martínez / ORC ID: 0000-0001-7111-4492, Researcher ID: X-3852-2019

ID 2do Coautor: Anahí Monserrat, Torres-Tinoco / ORC ID: 0000-0001-5625-8076, Researcher ID: T-5748-2018, arXiv: AnahiTinoco, CVU CONACYT ID: 236283

ID 3er Coautor: Ricardo Miguel, Sánchez-Durán / ORC ID: 0000-0001-8742-2879, Researcher ID: G-1904-2019, CVU CONACYT ID: 972644

DOI: $10.35429 / J P D .2019 .8 .3 .27 .34$ Recibido 01 de Abril, 2019; Aceptado 28 de Junio, 2019

\section{Resumen}

En este artículo se muestra el uso de buenas prácticas del modelo de calidad CMMI como parte de la metodología de trabajo aplicada en el aula y con el modelo de enseñanza aprendizaje basados en proyectos. El primer paso fue la definición de la metodología de trabajo, como ya se ha mencionado adoptando buenas prácticas del modelo de calidad CMMI. Se continua con la definición e integración del órgano colegiado quienes desempeñan el rol de líderes expertos en sus áreas de trabajo y definen las características que forma el proyecto integrador. Por último, se formaron los equipos de trabajo para la implementación de la metodología en un escenario real apoyándose con herramientas tecnológicas para facilitar la gestión de este. La contribución fue proponer una metodología de trabajo que ofrezca una mejora en la experiencia del proceso enseñanza-aprendizaje, incremente el nivel de conocimientos y que favorezca a la inclusión laboral de los egresados.

CMMI, Aprendizaje basado en proyectos, Modelos de calidad

\begin{abstract}
This article shows the use of good practices of the CMMI quality model as part of the work methodology applied in the classroom and with the project-based teachinglearning model. The first step was the definition of the work methodology, as already mentioned adopting good practices of the CMMI quality model. It continues with the definition and integration of the collegiate body who play the role of expert leaders in their work areas and define the characteristics that form the integrating project. Finally, work teams were formed for the implementation of the methodology in a real scenario, supported by technological tools to facilitate its management. The contribution was to propose a work methodology that offers an improvement in the teachinglearning process experience, increases the level of knowledge and that favors the labor inclusion of graduates.
\end{abstract}

CMMI, Project-based learning, quality models

Citación: LÓPEZ-SEGURA, Teresita de Jesús, NACHEZ-MARTÍNEZ, Wendy Denisse, TORRES-TINOCO, Anahí Monserrat y SÁNCHEZ-DURÁN, Ricardo Miguel. CMMI aplicado en el aula: aprendizaje basado en proyectos. Revista de Didáctica Práctica. 2019. 3-8: 27-34.

*Correspondencia al Autor (Correo Electrónico: tlopez@utleon.edu.mx)

$\dagger$ Investigador contribuyendo como primer autor. 


\section{Introducción}

En el reporte Future of the classroom, publicado por Google for Education, especifica 8 tendencias educativas donde los especialistas concluyen que la educación está evolucionando para que los estudiantes estén más conectados en sus vidas, colaboren más en clase y estén mejor preparados para desempeñar actividades profesionales, resaltando que, para lograr el éxito educativo, la tecnología y la pedagogía deben trabajar juntas, bajo estas tendencias se realizó esta investigación.

Otra perspectiva que nos abona, es el artículo "The Main Course, Not Dessert" de John Larmer y John R. Mergendoller, donde mencionan que no es lo mismo trabajar con proyectos que trabajar por proyectos. El aprendizaje basado en proyectos es un plato principal rico en contenidos curriculares y en competencias clave que son demandadas por la sociedad y la industria actual.

Al hablar de aprendizaje no se debe perder de vista las características del aprendizaje basado en proyectos, el cual permite a los alumnos adquirir los conocimientos y competencias mediante la elaboración de proyectos que dan respuesta a problemas de la vida real, también puede ser clasificado como aprendizaje activo.

El valor agregado en esta investigación es la implementación de buenas practicas del modelo de calidad CMMI en la metodología utilizada para el desarrollo de los proyectos integradores.

Hoy en día, el CMMI es un modelo importante para la mejora de procesos y el desarrollo de software. Las empresas que lo implementan experimentan una mayor productividad y calidad, una mejor duración del ciclo de vida y presupuestos más precisos y predecibles. Este es el motivo principal por el cual se incluyeron las practicas del modelo en la metodología de trabajo, permitiendo al alumno adquirir las competencias profesionales propias de la carrera y además adquirir competencias de modelos de calidad internacionales demandas por la sociedad y la industria actual.
El primer paso fue la definición de la metodología de trabajo, como ya se ha mencionado adoptando buenas prácticas del modelo de calidad CMMI. Se continua con la definición e integración del órgano colegiado quienes desempeñan el rol de líderes expertos en sus áreas de trabajo y definen las características que forma el proyecto integrador. Por último, se formaron los equipos de trabajo para la implementación de la metodología en un escenario real apoyándose con herramientas tecnológicas para facilitar la gestión de este. La contribución fue proponer una metodología de trabajo que ofrezca una mejora en la experiencia del proceso enseñanza-aprendizaje, incremente el nivel de conocimientos y que favorezca a la inclusión laboral de los egresados.

\section{Planteamiento del problema}

Los alumnos de la carrera Multimedia y Comercio Electrónico, de la Universidad Tecnológica de León, desarrollan proyectos integradores en dos periodos, en tercer y quinto cuatrimestre. Los proyectos integradores solían atender a escenarios simulados y algunos fueron desarrollados para clientes reales, sin embargo, se detectó en generaciones pasadas que el proceso de aprendizaje de los alumnos se tornaba confuso y descontrolado, permitiendo una carente aplicación de conocimientos en los proyectos integradores sin logros significativos. Sumando que los egresados iniciaban sus actividades en el mundo laboral, con poca o nula confianza en sus habilidades y competencias profesionales.

Posterior, durante el periodo de estadías, y la cercanía que se daba con empresas de la región, se obtuvo algunas de las recomendaciones de empresarios respecto al perfil de los alumnos por egresar, estas fueron: incrementar el nivel de autonomía, ser proactivo, mejorar la comunicación, trabajo en equipo y liderazgo.

La situación detectada requería una mejora en el proceso de desarrollo de proyectos integradores que permitiera un aprendizaje significativo y a su vez que brindara al alumno la adquisición de competencias básicas, genéricas y especificas propias de su perfil profesional y que pudieran satisfacer las necesidades actuales de la sociedad e industria. 


\section{Justificación}

En los últimos años, el estado de Guanajuato ha presentado un crecimiento importante en la industria, mucho de esto, se debe a la llegada de empresas de la rama automotriz, mismas que demandan capital humano preparados para los nuevos retos, y entre estos, se identifica la integración de la industria 4.0 en la gestión y producción de las empresas, por ejemplo, en la automatización, operaciones basadas o soportadas por el internet de las cosas y la alta especialización de sus trabajadores.

A partir de las necesidades detectadas, tanto de la industria como del proceso enseñanza-aprendizaje, se propuso los cambios en la metodología de desarrollo de proyectos integradores, atendiendo al perfil de egreso de la carrera y a las necesidades actuales de la industria, y profundizar los conocimientos sobre los modelos de calidad y mejora continua, además de ofrecer a los alumnos formación integral en el desarrollo y la gestión de proyectos, potencializando su inclusión laboral.

\section{Objetivos}

\section{Objetivo general}

Mejorar el aprendizaje de los estudiantes a través del modelo de enseñanza aprendizaje basado en proyectos, ejecutando buenas prácticas del modelo de mejora CMMI que permitan desplazar el centro del conocimiento memorístico a la aplicación del conocimiento en escenarios reales

\section{Objetivos específicos}

- Integrar conocimientos y habilidades de varias áreas a través de proyectos multidisciplinares.

- Desarrollar autonomía que propicie la investigación y la búsqueda de información, y en este contexto desarrollar la capacidad para discernir que información es fiable y cual no lo es.

- Sustentar a través de procesos el logro de objetivos y mejorar el rendimiento de los recursos.
- Fomentar el trabajo en equipo, mantener la comunicación efectiva y asignar las actividades y recursos en tiempo y forma.

- Disminuir los defectos de los productos generados logrando una mayor satisfacción de los beneficiarios.

\begin{abstract}
Alcance
Esta investigación se enfocó en implementar buenas prácticas del modelo de mejora CMMI durante el desarrollo de proyectos integradores de los alumnos de quinto cuatrimestre de la carrera Multimedia y Comercio Electrónico de la Universidad Tecnológica de León, durante el periodo enero-abril 2019.
\end{abstract}

\section{Marco de Referencia}

\section{CMMI (Capability Maturity Model Integration)}

De acuerdo con un extracto del libro publicado por Pearson Educación, S.A. en el año 2009 con el título CMMI, Guía para la integración de procesos y la mejora de productos, CMMI (Capability Maturity Model Integration) es:

[...] un modelo de madurez de mejora de los procesos para el desarrollo de productos y de servicios. Consiste en las mejores prácticas que tratan las actividades de desarrollo y de mantenimiento que cubren el ciclo de vida del producto, desde la concepción a la entrega y el mantenimiento. El modelo de CMMI tiene cinco niveles de madurez, designados del 1 al 5, como se muestran en la Figura 1.

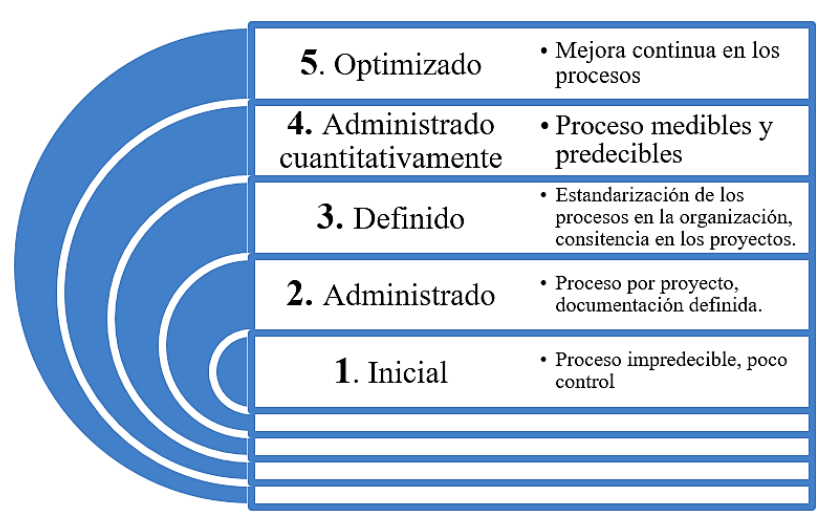

Figura 1 Niveles de Madurez de CMMI 
Cada nivel trae consigo áreas clave de proceso, definamos un área de proceso como un conjunto de practicas relacionadas en una zona que, cuando se implementan en conjunto, satisfacen un conjunto de objetivos importantes para realizar mejoras significativas en la materia. Se pueden indicar en cuatro categorías:

- Gestión de procesos.

- Gestión de proyectos.

- Ingeniería.

- Apoyo.

Cada área de proceso se define con un conjunto de objetivos y prácticas. Hay dos categorías:

- Metas y practicas genéricas: Son una parte de cada área de proceso.

- Metas específicas y practicas: son específicos de una determinada área de proceso.

Este modelo fue creado por la CarniegeMellon University con la finalidad de unificar la gran cantidad de modelos de desarrollo de software creados por otras organizaciones a lo largo de los años. De ahí que actualmente sea uno de los modelos más usados dentro de la industria del software.

Es importante mencionar, con la implementación del modelo CMMI es factible la disminución o eliminación de retrabajo, pero además aumenta la fiabilidad en lo que se refiere a la predicción de costos, incrementa la reutilización de productos y procesos, pero sobre todo disminuye costos debido a las múltiples evaluaciones y programas de mejoras de proceso.

\section{Características del aprendizaje basado en proyectos}

Un aprendizaje basado en proyecto se caracteriza por:

- Objetivos de aprendizaje planeados en un proyecto y que derivan de las competencias clave de las asignaturas involucradas.
- Requiere pensamiento crítico, resolución de problemas, colaboración, y, además, diversas formas de comunicación.

- Incluye la evaluación y reflexión, los alumnos deben aprender a evaluar y ser evaluados para mejorar la calidad de los productos desarrollados.

- Algunas ocasiones se invita a audiencia para medir los logros adquiridos por los alumnos y además brindar recomendaciones de gestión y ejecución del proyecto.

\section{Metodología}

Definición de la metodología de trabajo implementando buenas prácticas del modelo de mejora CMMI. Se tomo como base el modelo incremental de gestión de proyectos, el cual consiste en un crecimiento progresivo de la funcionalidad. En otras palabras, el producto va evolucionando con cada una de las entregas previstas hasta que se amolda a lo requerido por el cliente. Las fases definidas fueron:

1. Inicio

2. Planeación

3. Ejecución
a. Análisis
b. Diseño
c. Construcción
d. Pruebas e Implementación

4. Monitoreo y Control

5. Cierre

Dentro de esta metodología se implementaron las siguientes prácticas genéricas del modelo CMMI:

- GP 2.4 Asignar responsabilidad.

- GP 2.7 Identificación y participación de las partes interesadas.

- GP 2.8 Supervisar y controlar el proceso.

- GP 3.1 Establecer un proceso definido.

- GP 3.2 Recopilar información de mejora.

- GP 5.2 Corregir causas de los problemas 
permiten:

Con las practicas anteriores nos

- Identificar la capacidad de realizar las actividades, en aspectos materiales, capital humano, tecnológico, de gestión y de conocimiento.

- Evaluar el nivel de compromiso de los involucrados para garantizar que el procedimiento establecido se seguirá y se mantendrá.

- Planear actividades, dar seguimiento y tomar medidas correctivas cuando sea necesario.

Además de las practicas genéricas, se agregaron varias prácticas de áreas de procesos específicas como: (DAR).

Análisis y resolución de decisiones

- SP 1.3 Identificar soluciones alternativas.

- SP 1.5 evaluar alternativas.

Proyecto de Monitoreo y Control.

- SP 1.3 Supervisar los Riesgos del proyecto

Planificación de proyectos.

- SP 1.1 estimar el alcance del proyecto.

- SP 2.1 Establecer el presupuesto y el calendario.

- SP 2.2 Identificar los riesgos del proyecto.

- SP 2.3 Plan de Gestión de Datos.

- SP 2.4 Plan de recursos del proyecto.

- SP 2.5 Plan de conocimientos y habilidades necesarios.

- SP 3.2 conciliar el trabajo y en los niveles de recursos.

Desarrollo de requerimientos.

- SP 1.2 Establecer los requisitos del cliente.

- SP 3.3 Analizar los requisitos.
Solución técnica.

- SP 2.1 Diseño del producto o componente del producto.

- SP 2.3 Las interfaces de diseño utilizando criterios.

- SP 3.1 implementar el diseño.

- SP 3.2 Desarrollar Documentación de soporte del producto.

El sumar las practicas del modelo CMMI a la metodología planteada nos permite fomentar el trabajo en equipo, establecer procesos de trabajo, mantener canales de comunicación claros y efectivos, fomenta la distribución de esfuerzos, es decir, la asignación de actividades y recursos, además de integrar conocimientos y habilidad de los involucrados.

\section{Definición e integración del órgano colegiado}

Se integran actividades desarrolladas en las asignaturas Multimedia II, Desarrollo de Sitios Web para Comercio Electrónico, Administración de proyecto, Contabilidad, Expresión Oral y Escrita e Integradora II, se gestionaron reuniones de trabajo para definir objetivo del proyecto, alcance y entregables por asignatura y por parcial, permitiendo a los alumnos adquirir y demostrar el nivel de competencia adquirida a través de prácticas que abonan al proyecto integrador, y en caso de detectar que dicho nivel no es suficiente el docente diseñaba estrategias para lograr la competencia en el alumno.

\section{Implementación de la metodología}

Durante el cuatrimestre, se siguieron las actividades definidas en la metodología de trabajo sumando las buenas prácticas del modelo de mejora CMMI, como:

- Identificar cuáles son los objetivos de negocio a atender.

- Identificar agentes de cambio en su equipo de trabajo y externos, definir roles y responsabilidades. 
- Trabajo colaborativo, ayudar a ejecutar su trabajo de manera correcta, identificar dependencia de actividades y medir el impacto.

- Tomar conciencia del esfuerzo requerido tanto en tiempo como en recursos.

- Monitoreo y control de los procesos, que consiste en realizar la verificación del cumplimiento de los procesos y actividades definidas, así como la generación de los productos de trabajo, permitiendo identificar desviaciones y definir las acciones correctivas correspondientes.

\section{Uso de herramientas tecnológicas en la gestión del proyecto}

- Como parte de la gestión del proyecto se usaron herramientas como el repositorio de archivos Google Drive y plataformas de gestión de proyectos como Workep y TeamWork, las cuales permitieron tener un mejor control del trabajo, algunas de las ventajas que brindaron, fueron:

○ Facilidad en la ejecución de metodologías, pues son herramientas flexibles y autogestionables con interfaz gráfica amigable y con una rápida curva de aprendizaje.

- Monitoreo del progreso, permite hacer un seguimiento al trabajo que se está realizando en cualquier momento.

- Una característica importante que se identificó al usar estas herramientas fue, que permiten identificar el flujo de trabajo de forma gráfica, disminuyendo el nivel de confusión de los colaboradores.

\section{Resultados}

Los impactos más sobresalientes de esto proyecto de investigación fueron:

- La metodología permitió integrar eficazmente los conocimientos y los esfuerzos de los alumnos en el desarrollo del proyecto integrador. Además, se puede incluir que permitió una mejor comunicación y colaboración del órgano colegiado.
- Se fomentaron actividades de investigación para el sustento y definición del proyecto a desarrollar.

- En el desarrollo del proyecto se observó la importancia de una comunicación efectiva en los equipos de trabajo y con el beneficiario, para el logro de los objetivos del proyecto.

- Se redujo el descontrol y desconocimiento del plan de trabajo de los proyectos integradores, a través de estrategias de comunicación y reuniones para la toma de acuerdos.

- Se observo en los productos resultantes de cada proyecto, una mejora en la calidad, tanto en las aplicaciones desarrolladas como los productos generados en la gestión del proyecto.

Los alumnos lograron adquirir buenas prácticas del modelo de mejora CMMI, incrementando sus capacidades y su experiencia profesional, y en este contexto se impulsa el perfil de egreso de los alumnos pues implementan prácticas de un modelo de mejora reconocido a nivel mundial.

\section{Agradecimientos}

Al equipo de profesores de la carrera Multimedia y Comercio Electrónico de la UTL, por su apoyo, contribución y su constancia en la formación integral de nuestros estudiantes.

A la Universidad Tecnológica de León por el apoyo con las horas de investigación asignadas para que este proyecto pudiera desarrollarse

\section{Conclusiones}

El aprendizaje basado en proyectos y la metodología planteada, permitieron a los alumnos incrementar el nivel de competencias básicas, genéricas y especificas del perfil de egreso, como aplicar técnicas de administración, diseño y desarrollo de aplicaciones, para ofertar servicios de innovación tecnológica de productos multimedia. 
"Durante el cuatrimestre enero-abril 2019, tuve la oportunidad de realizar un proyecto que reunía todas las materias de mi área, integradora II. Dicho proyecto fue gestionado con prácticas del modelo de calidad CMMI por lo que permitió tener una adecuada gestión de tiempo, de recursos y demás.

Las técnicas implementadas para el proyecto me parecieron muy efectivas puesto que daba pauta a entregas en tiempo y forma, con dichas prácticas se daba información clara de lo que se realizaba y como se estaba dando solución a situaciones que fueran externas al proyecto, por lo que la organización fue la clave para un adecuado seguimiento de estas al estar buscando la mejora en cada uno de los procesos realizados.

Además, cuando se está en el aula es complicado comprender desde donde debes partir para comenzar con un proyecto, que cosas debes tomar en cuenta para poder tener éxito en la entrega y cumplir con las características o requisitos que son solicitados en periodos tan cortos.

Por esa razón considero que todas las actividades solicitadas por integradora II tenían como base planificar adecuadamente el proyecto y encontrar áreas de riesgos para con ello identificar la solución a cada uno de los escenarios que pudieran presentarse, no obstante, el tener un control de recursos, involucrados y datos ayudó a que se mejorará aquellas actividades o prácticas que no estaban siendo adecuadas mediante el objetivo y planes realizados en los mismos.

Finalmente, cuando entre en un área laboral me hizo comprender la utilidad de la gestión del proyecto integrador, puesto que te abre el panorama a realizar tus actividades con el objetivo de entregar calidad en un proyecto, de identificar todo aquello que impide que el proyecto avance e implementar lo que ya practicaste, tanto la organización de tus tiempos, tus tareas como la comunicación de aquello que estás detectando que falta o que es correcto.
Por dicha razón las competencias que se adquieren en el aula te dan un plus en un ambiente laboral cuando cumpliste con cada una de las prácticas que te fueron solicitadas puesto que ya identificas aquellos errores que se están cometiendo y las mejoras que son necesarias llevar a cabo para dar solución a cualquier problemática", son los comentarios de Wendy Denisse Nachez Martínez, quien fue alumna de la carrera y formo parte de los proyectos integradores.

Las practicas recomendadas por el modelo CMMI son muchas y algunas requieren un alto nivel de esfuerzo para su ejecución, siempre debe evaluarse si estas prácticas agregan valor al producto $\mathrm{o}$ proceso desarrollado. Actualmente se ha liberado una versión CMMI 2.0, el cual promete ser más dinámico, ligero y adaptable además implementa prácticas ágiles, esta versión podría mitigar algunas desventajas de la versión anterior.

\section{Referencias}

Chrissis, M. B., Konrad, M., \& Shrum, S. (2009). CMMI, Guía para la integración de procesos y la mejora de productos. España: Pearson Educación, S.A.

CMMI Institute. (20 de Agosto de 2019). CMMI Institute. Obtenido de CMMI Institute: https://cmmiinstitute.com/cmmi/intro

Google For Education. (2019). Google For Education. Obtenido de Google For Education: $\mathrm{http} / / /$ services.google.com/fh/files/misc/future_ of_the_classroom_emerging_trends_in_k12_ed ucation.pdf?utm_source=web\&utm_campaign $=$ FY19-Q2-global-demandgen-website-other-

futureoftheclassroom

Larmer, J., \& Mergendoller, J. R. (2011). Buck Institute for Education . Obtenido de Buck Institute for Education : http://files.ascd.org/pdfs/onlinelearning/webinar s/webinar-handout1-10-8-2012.pdf

Roberto, H. S. (2018). Metodología De La Investigación: Las rutas cuantitativa, cualitativa y mixta. D.F.: McGraw-Hill. 
Software Guru. (abril de 2014). CMMI + SCRUM, no CMMI VS SCRUM! Obtenido de https://sg.com.mx:

https://sg.com.mx/sgvirtual/7/sessions/cmmiscrum-no-cmmi-vs-scrum

Software Guru. (2019). CMMI v2.0: Más dinámico, ligero y adaptable. Obtenido de https://sg.com.mx/:

https://sg.com.mx/buzz/ponencias/sg-virtual14/cmmi-v20-mas-dinamico-ligero-y-adaptable

UNESCO . (2019). Organizacion de las Naciones Unidas para la Educación, la Ciencia y la Cultura . Obtenido de Organizacion de las Naciones Unidas para la Educación, la Ciencia y la Cultura : https://es.unesco.org/themes/ticeducacion 